\title{
Einführung: Ursachen und Folgen ökonomischer Desintegration im 20. Jahrhundert
}

\author{
von Jens Beckert und Carsten Burhop
}

\begin{abstract}
„Deutschland lebt in der Europäischen Union in einer Schicksalsgemeinschaft. Ihr verdanken wir Jahrzehnte des Friedens, des Wohlstandes und des Einvernehmens mit unseren Nachbarn. “1 „Niemand sollte glauben, dass ein weiteres halbes Jahrhundert Frieden und Wohlstand in Europa selbstverständlich ist. Es ist es nicht. Deshalb sage ich: Scheitert der Euro, dann scheitert Europa.“2 „Wir müssen uns jetzt aufmachen, das nachzuholen, was vor 20 Jahren bei der Gründung der Wirtschafts- und Währungsunion durch den Vertrag von Maastricht noch nicht möglich war: die Wirtschafts- und Währungsunion politisch $\mathrm{zu}$ vollenden. ${ }^{\text {”3 }}$
\end{abstract}

Schenkt man der Krisenrhetorik politischer Entscheidungsträger Glauben, dann folgt auf die Desintegration der Eurozone auch die wirtschaftliche und politische Desintegration Europas, eine Verarmung des Kontinents und letztlich Krieg zwischen heute in Frieden, Freiheit und Wohlstand lebenden Nationen. Verhindert werden kann dies nur, indem man die Desintegration des Euro verhindert und den Weg in eine vollständige wirtschaftliche und politische Integration beschreitet - ein Plan, der bereits Ende der 1980er Jahre von der Regierung Kohl/Genscher erfolglos verfolgt worden ist. ${ }^{4}$ In Paris und London wurde damals zwar die Abschaffung der D-Mark zur Begrenzung der wirtschaftlichen Macht Deutschlands begrüßt, eine weitergehende politische Integration wurde jedoch abgelehnt, weil sie nur unter dem Primat eines deutschen Europas funktionieren könne und anderenfalls $\mathrm{zu}$ Instabilität führe. ${ }^{5}$ Die damalige Bundesregierung erkannte zwar, dass eine Währungsunion ohne politische Union keinen Sinn ergebe, aber sie war auch davon überzeugt, dass eine Veränderung des europäischen Projekts von Frankreich ausgehen müsse. ${ }^{6}$ Gleichwohl dominierte noch bei der physischen Einführung des Euro am

1 Regierungserklärung von Bundeskanzlerin Dr. Angela Merkel zu den Hilfen für Griechenland am 5. Mai 2010, http://www.bundesregierung.de/Content/DE/Regierungserklaerung/2010/2010-05-05-merkel-erklaerung-griechenland.html.

2 Regierungserklärung von Bundeskanzlerin Dr. Angela Merkel zum Europäischen Rat und zum Eurogipfel am 26. Oktober 2011, http://www.bundesregierung.de/Content/DE/Regierungserklaerung/2011/2011-10-27-merkel-eu-gipfel.html.

3 Regierungserklärung von Bundeskanzlerin Dr. Angela Merkel am 27. Juni 2012 zum Europäischen Rat am 28. und 29. Juni 2012 in Brüssel, http://www.bundesregierung.de/ Content/DE/Regierungserklaerung/2012/2012-06-27-bkin.html.

4 Hans-Peter Schwarz, Helmut Kohl. Eine politische Biographie, München 2012, S. 560.

5 Margret Thatcher, Downing Street No. 10. Die Erinnerungen, Düsseldorf 1993, S. 1034.

6 Schwarz, Helmut Kohl, S. 807. 
1. Januar 2002 großer Optimismus beim überzeugten Europäer Helmut Kohl. Er war sich seinerzeit sicher, dass „in fünf oder sechs Jahren [...] auch die Briten mit dem Euro zahlen [werden]. "Zudem wagte er einen weiteren Ausblick, in dem er prognostizierte, dass es 2012 die einheitliche Währung auch in Zürich geben werde. ${ }^{7}$

Diese Prognose des ehemaligen Bundeskanzlers ist offensichtlich nicht eingetreten - immer noch gibt es das Pfund Sterling und den Schweizer Franken. Dies wirft nicht nur die Frage nach der Prognosefähigkeit von Bundeskanzlern auf: Folgt auf einen Prozess ökonomischer Desintegration stets eine wirtschaftliche, politische, gesellschaftliche und sogar militärische Krise, wie es die Aussagen von Bundeskanzlerin Angela Merkel nahelegen? Dieser Frage gingen die Referenten, Diskutanten und Teilnehmer einer Tagung über die „Ursachen und Folgen ökonomischer Desintegration im 20. Jahrhundert" im April 2012 nach. Weder können im Rahmen einer Tagung noch im Umfang eines Zeitschriftenbandes alle Desintegrationsprozesse in all ihren Facetten beleuchtet werden. Bei der Auswahl standen - in Anbetracht der derzeitigen Situation in Europa Desintegrationsprozesse von Währungsräumen im Vordergrund: das Ende des Golddevisenstandards in den 1930er Jahren, die Auflösung der Sterlingzone während der 1960er Jahre und der anschließende Verfall des Weltwährungssystems von Bretton Woods. Dem steht lediglich ein realwirtschaftlicher Fall gegenüber, nämlich das Ende des Rates für gegenseitige Wirtschaftshilfe, dem nach dem Fall des Eisernen Vorhangs abgewickelten sozialistischen Pendant zur Europäischen Gemeinschaft.

Dieser Schwerpunkt des vorliegenden Themenheftes schließt eng an aktuelle wirtschaftshistorische wie auch gesellschaftspolitische Debatten an. So schilderte kürzlich der amerikanische Wirtschaftshistoriker Barry Eichengreen die Ablösung des britischen Pfundes durch den amerikanischen Dollar als internationale Leitwährung und diskutierte, ob der Dollar durch den Euro oder den chinesischen Yuan abgelöst werden könnte. Er kommt zu dem Schluss, dass der Euro derzeit nicht zur neuen Weltwährung werden könne, weil er eine Währung ohne Staat sei. ${ }^{8}$ Der amerikanische Ökonom Douglas Irwin zeigte, dass die Weltwirtschaftskrise der 1930er Jahre durch einen nicht auflösbaren Widerspruch zwischen offenem Welthandel und fixierten Währungen verschärft worden sei. Damals mussten die Regierungen entweder den Freihandel oder den Goldstandard opfern, um den Weg aus der Depression zu beschreiten. ${ }^{9} \mathrm{Da}$ innerhalb der Eurozone jedoch weder der Freihandel aufgegeben noch die Wechselkurse verändert werden können, bleibe nur der Weg der inneren Abwertung, um die gegenwärtige Rezession in den Krisenstaaten zu überwin-

7 Helmut Kohl, Wir haben für die Zukunft das Notwendige getan, in: FAZ, 31.12.2001, S. 14.

8 Barry Eichengreen, Exorbitant Privilege. The Rise and the Fall of the Dollar and the Future of the International Monetary System, Oxford 2011, S. 130.

9 Douglas A. Irwin, Trade Policy Disaster. Lessons from the 1930s, Cambridge, MA 2012. 
den. Die Ursachen und vor allem die Wirkungen dieser Politik sind jedoch äußerst umstritten. ${ }^{10}$

In der gegenwärtigen Krise werden die grundsätzlichen Vor- und Nachteile ökonomischer Integration erneut diskutiert. Die Abschaffung von Handelsbarrieren sowie die Freizügigkeit von Arbeitskräften und Kapital führen zu einer besseren Allokation der Produktionstätigkeit. Die Güter werden nun eher dort produziert, wo sie relativ am besten hergestellt werden können. Die Produktionsfaktoren Arbeit und Kapital strömen im Fall integrierter Märkte ebenfalls potentiell zu den Orten, an denen sie am besten eingesetzt werden können. Eine gemeinsame Währung erleichtert diese Bewegungen, weil Transaktionskosten entfallen und weil die Unsicherheit bezüglich des zukünftigen Wechselkurses entfällt. In der Praxis zeigt sich allerdings, dass nicht alle Güter und Produktionsfaktoren flexibel sind. Eine Fabrik kann nicht einfach abgerissen und anderenorts wieder aufgebaut werden, und Arbeitskräfte können nicht ohne Weiteres Sprachbarrieren überwinden. Der ökonomische Druck, der aus dem Zwang zur vollständigen Flexibilisierung von Güter- und Faktormärkten resultiert, muss dann auf anderem Wege abgebaut werden. Traditionelle Verfahren waren Subventionen und periodische Veränderungen politisch festgelegter Wechselkurse. ${ }^{11}$ Im Golddevisenstandard, im Rat für gegenseitige Wirtschaftshilfe, im Weltwährungssystem von Bretton Woods und in der Europäischen Gemeinschaft sowie im Europäischen Währungssystem standen diese traditionellen Methoden zur Verfügung. Demgegenüber erlaubt die Gestaltung der Eurozone ihren Einsatz nicht, so dass man neue Lösungen finden muss. Findet man adäquate Lösungen nicht innerhalb der bestehenden Ordnung, dann könnte sich ein neues Arrangement bilden. Radikale Lösungen werden derzeit in der Öffentlichkeit diskutiert. Beispielsweise stellte ein Journalist des britischen Economist unlängst fest, dass

financial contagion may have been contained for now by the European Central Bank, but political contagion is a growing risk. Unless growth returns the day may come when one country decides, as with the gold standard in the 1930s, that the pain of adjustment outweights the risks of leaving. ${ }^{12}$

Neben dem Austritt eines krisengeplagten Landes aus der Eurozone wird in der Öffentlichkeit tatsächlich auch ein Austritt Deutschlands erwogen:

10 Vgl. u.a. Wolfgang Streeck, Gekaufte Zeit. Die vertagte Krise des demokratischen Kapitalismus, Berlin 2013.

11 Vgl. u.a. Herbert Giersch u.a., The Fading Miracle. Four Decades of Market Economy in Germany, Cambridge 1992, Kap. 4B.

12 Kolumne Charlemagne. Euro Wobbles, in: The Economist, 13.4.2013, S. 30. 
Die erstbeste Lösung wären Euro-Bonds, die zweitbeste ein Euro-Austritt Deutschlands. Die schlechteste Lösung wäre es, wenn ein Krisenland wie Italien den Euro verlassen würde, denn das würde zu einer ungeordneten Auflösung der EU führen. ${ }^{13}$

In seinem Beitrag zum Ende des Golddevisenstandards, der die Weltwährungsordnung im Zeitraum zwischen dem Ersten und Zweiten Weltkrieg dominierte, legt Matthias Morys dar, dass sich zeitgenössische Akteure ein Ende dieses Währungssystems nicht vorstellen konnten und dass sie der nach und nach erfolgten Desintegration in mehrere Währungsblöcke in den 1930er Jahre eine krisenverschärfende Wirkung zuschrieben. Ohne den Zusammenbruch des Golddevisenstandards, so die damalige Auffassung, wäre die Weltwirtschaftskrise weniger tief und kürzer gewesen. Erst fünf Dekaden nach dem Systemzusammenbruch entstand eine neue herrschende Meinung hinsichtlich seiner Folgen. Seitdem glaubt man, dass insbesondere der Abschied von den goldenen Fesseln zur relativ raschen Erholung der Weltwirtschaft beigetragen hat. Nachdem ein Land die Goldbindung seiner Währung aufgegeben hatte, konnte es die binnenwirtschaftliche Geld- und Kreditversorgung verbessern und die Exportchancen durch Abwertung steigern. Der zweite Effekt war zwar nur ein vorübergehender, weil er durch die Abwertungen oder Zollerhöhungen anderer Länder bald ausgeglichen wurde, aber die steigende inländische Geldversorgung führte zu steigenden Preisen, so dass Schuldner durch Inflation entlastet wurden. Zudem erleichterte die lockere Kreditpolitik Investitionen.

Im Rückblick ist zudem offensichtlich, dass die Desintegration alternativlos auf die fehlerhafte Integration folgen musste. Drei strukturelle Probleme arbeitet Morys heraus: Erstens bedeutete der deutliche Anstieg des Preisniveaus während des Ersten Weltkrieges, dass ein signifikant gewachsenes volkswirtschaftliches Transaktionsvolumen auf einer unveränderten Goldbasis stand. Die Glaubwürdigkeit, dass Zentralbanken ihre zahlreichen Banknoten in Gold umtauschen könnten, war somit gering. Zweitens war eine Senkung des allgemeinen Preisniveaus durch kostensenkende Lohnkürzungen der Unternehmen kaum noch möglich infolge der in vielen Ländern gewachsenen politischen Partizipation von Arbeitnehmern. Drittens hatte die Verwandlung vom Goldstandard der Vorkriegszeit zum Golddevisenstandard der Zwischenkriegszeit die weltwirtschaftliche Vernetzung der Nationalökonomien erhöht, weil nun jede am System teilnehmende Zentralbank einen Teil ihrer Banknoten mit den Banknoten anderer Zentralbanken deckte. Wenn eine Zentralbank ihre Banknoten nicht mehr in Gold einlösen konnte oder wollte, dann hatte dies unmittelbar Auswirkungen auf die Deckungsfähigkeit der anderen Zentralbanken. Eine Deckungskrise in einem wichtigen Land konnte so andere Länder rasch anstecken.

13 George Soros in einer Rede an der Universität Frankfurt am 9. April 2013. Zit. n. Sebastian Jost u. Holger Zschäpitz, Soros wirbt für Euro-Austritt, in: Die Welt, 11.4.2013, S. 17. 
Banken- und Fiskalkrisen in den Vereinigten Staaten und Deutschland lösten den Zusammenbruch aus. Im Verlauf der Weltwirtschaftskrise nahmen Unternehmenskonkurse und Arbeitslosigkeit in Deutschland zu, die Steuereinnahmen fielen, die staatlichen Transferzahlungen stiegen und die Defizite der öffentlichen Haushalte nahmen zu. Vermehrt sah sich der durch die Wirtschaftskrise geschwächte deutsche Bankensektor von einer Staatsfinanzkrise bedroht. Als dann Reichskanzler Brüning erklärte, dass Deutschland seinen Reparationsverpflichtungen - vorübergehend - nicht nachkommen könne, setzte ein run auf die deutschen Banken und die deutsche Währung ein. Die deutsche Regierung reagierte mit Kapitalverkehrskontrollen und Devisenbewirtschaftung. Daraufhin konnte zwar die Reichsbank die inländische Geldmenge hemmungslos ausdehnen und damit die Banken retten, aber ausländische Gläubiger konnten ihre Forderungen gegenüber deutschen Banken und Geschäftspartnern nicht mehr realisieren. So entstand rasch eine Liquiditätskrise am Weltfinanzplatz London. Die britische Regierung und die Bank of England reagierten mit einer Abwertung des Pfundes. Die Weltwährungskrise war da.

Nach dem Zweiten Weltkrieg wurde das Währungssystem der freien Welt auf der Weltwährungskonferenz von Bretton Woods neu justiert. Grundsätzlich feste, aber in Sonderfällen adjustierbare Wechselkurse sollten den Welthandel fördern. Darüber hinaus wurden Währungsverbünde und Zahlungsverkehrsabkommen, beispielsweise die Sterlingzone und die Europäische Zahlungsunion, geschaffen, weil viele Länder ihren Außenhandel noch nicht in der internationalen Leitwährung, dem US-Dollar, abwickeln konnten. Eng begrenzte Dollarbestände, eine hohe Auslandsverschuldung und großer Importbedarf waren Gründe für den Erhalt der sogenannten Sterlingzone nach dem Zweiten Weltkrieg. Catherine R. Schenk beschreibt in ihrem Aufsatz die vier Lebensphasen der Sterlingzone: die Phase des geschlossenen Systems (1946 - 1952) wurde von den Phasen der langsamen Liberalisierung (1952-1958) und der Diversifizierung der Wirtschaftsbeziehungen (1959-1967) und schließlich von der Phase der verwalteten Auflösung (1968-1972) abgelöst. Ähnlich der Europäischen Zahlungsunion zielte auch die Sterlingzone in ihren ersten beiden Lebensphasen darauf, den Bedarf an Dollarscheinen im Außenhandel der Mitgliedsländer zu begrenzen, indem man sich darauf einigte, dass die Verrechnung innerhalb der Zone in Pfund Sterling vorgenommen wurde, und indem man vereinbarte, nur einen kleinen Teil des Außenhandels mit Nicht-Mitgliedern abzuwickeln. Ab 1952 öffneten sich einzelne Länder nach und nach für den freien Welthandel, weil dies ihren eigenen wirtschaftlichen Interessen entsprach. Mit der beschleunigten Dekolonisierung Ende der 1950er Jahre gewannen zahlreiche Mitglieder der Sterlingzone ihre währungspolitische Autonomie und nutzen die neugewonnene Freiheit zunehmend zur Ausrichtung ihrer Währungspolitik an eigenen wirtschaftlichen Interessen. Infolgedessen nahm die Bedeutung des Pfundes als internationale Reservewährung ab. Viele Sterlingländer im asiatisch-pazifischen Raum orientierten sich zunehmend an den Vereinigten Staaten 
von Amerika. Gleichzeitig richtete sich die britische Wirtschaft seit den frühen 1960er Jahren deutlicher auf Westeuropa aus, so dass auch von dieser Seite die ökonomischen Vorteile der Sterlingzone geringer wurden. Die Erosion der Vorteilhaftigkeit nahm im Verlauf der 1960er Jahre zu. Zudem verursachte die überraschende und erhebliche Abwertung des Pfundes gegenüber dem Dollar im Jahre 1967 eine Vertrauenskrise. Länder, die ihre Währungsreserven in Pfund gehalten und auf einen festen Wert des Pfundes gegenüber dem Dollar vertraut hatten, sahen sich über Nacht einem erheblichen Wertverlust gegenüber. Lediglich die Dollarschwäche der Jahre um 1970 und die Tatsache, dass alternative Reservewährungen nicht verfügbar waren, ermöglichten eine langsame, durch Verträge geordnete Abwicklung der Sterlingzone.

Die Geschichte der Sterlingzone illustriert somit mehrere Aspekte, die auch in der gegenwärtigen Eurokrise relevant sind. Ein gemeinsamer Währungsraum muss den Teilnehmern wirtschaftliche Vorteile bringen, die Teilnehmer müssen auf die Stabilität des Arrangements vertrauen können und die Abwesenheit einer soliden Alternative stabilisiert ein bestehendes System.

Jenseits solcher ökonomischer Überlegungen weisen Carsten Burhop, Julian Becker und Max Bank hinsichtlich der Funktionsweise und des Zusammenbruchs des Weltwährungssystems von Bretton Woods, welches von den späten 1940 er bis in die frühen 1970er Jahre bestand, vor allem auf die Einbettung währungspolitischer Fragen in die allgemeine Außen- und Innenpolitik der jeweils beteiligten Staaten hin. Außerdem bemerken sie, dass die Struktur des Wechselkurssystems von Bretton Woods ein wesentliches Strukturdefizit des Golddevisenstandards erneut aufwies, weil die entscheidenden Währungspolitiker der 1940er Jahre unzureichende Erkenntnisse über die Krisenursachen der 1930er Jahre besaßen. Feste Wechselkurse und ein prinzipiell auf Gold beruhendes Währungssystem galten als förderlich für Stabilität und Prosperität der Weltwirtschaft. Im Gegensatz zum Golddevisenstandard versprachen im System von Bretton Woods nur noch die Vereinigten Staaten von Amerika den Umtausch ihrer Banknoten in Gold. Damit entstand zwar nach wie vor ein Problem, wenn die Menge der Dollarnoten schneller wuchs als die Goldmenge, aber es musste nur noch eine Währung in Gold umtauschbar sein, so dass eine größere Papiergeldmenge auf der Goldbasis aufgetürmt werden konnte. Es zeigte sich aber alsbald, dass der Geldbedarf der während des Wirtschaftswunders rasch wachsenden Weltwirtschaft zügig expandierte, wohingegen die verfügbare Menge des Währungsgolds mehr oder weniger stagnierte. Zudem nahmen die Goldreserven der Vereinigten Staaten fortlaufend ab, weil Kriege in Übersee und internationale Investitionen amerikanischer Unternehmen Zahlungsbilanzdefizite verursachten. Daher begannen die wichtigsten Staaten der westlichen Welt bereits 1961 - drei Jahre nach Wiedereinführung offener Devisenmärkte -, die Goldreserven der freien Welt zu poolen, um so die erodierende Goldbasis Amerikas zu stärken. Als jedoch wenige Jahre später die französische Regierung beschloss, den Pool zu verlassen sowie Dollar in Gold umzutauschen, war das Ende des Weltwährungssystems praktisch besiegelt. 
Nun folgten in immer kürzer werdenden Abständen immer größere Währungskrisen, die durch Gipfeldiplomatie, Notkredite, Abwertungen und Börsenschließungen bekämpft worden sind. Nach einigen Jahren der Krise hoben die Vereinigten Staaten im August 1971 die Goldeinlösungspflicht unilateral und ohne Abstimmung mit den „Partnerländern“ auf. Das auf Gold basierende Weltwährungssystem von Bretton Woods war gescheitert. Die aus tatsächlichen oder von den Märkten erwarteten makroökonomischen Ungleichgewichten resultierenden Wechselkurskrisen gehörten der Vergangenheit an, und die wirtschaftspolitischen Akteure konnten nun eine an den Interessen des Inlands ausgerichtete Politik betreiben. Insbesondere die von Goldfesseln und festen Wechselkursen befreiten nationalen Notenbanken konnten nun die von Fiskalkrisen und Ölschocks hart getroffenen Volkswirtschaften aus der Krise drucken - freilich um den Preis rasch steigender Inflationsraten.

Die gegenwärtig vertretene Auffassung von Regierung und Opposition hinsichtlich der Folgen einer Auflösung der Eurozone beinhaltet die Erwartung einer realwirtschaftlichen Desintegration, die infolge der Währungsdesintegration aufträte. Die Ursachen und Folgen einer realwirtschaftlichen Desintegration stellt André Steiner exemplarisch anhand des Rates für gegenseitige Wirtschaftshilfe (RGW), dem sozialistischen Pendant zur Europäischen Gemeinschaft, dar. Der RGW wurde 1949 geschaffen und sollte die außenwirtschaftlichen Aktivitäten der Planwirtschaften koordinieren. Befeuert wurde die realwirtschaftliche Integration des Ostblocks durch das vom Westen verhängte Handelsembargo. Die wirtschaftlichen Vorteile sollten maximiert werden, indem sich jedes Land auf den Export derjenigen Produkte konzentrierte, bei denen es den größten technischen und wirtschaftlichen Vorteil hatte. Unterlaufen wurde diese Politik jedoch durch die Industrialisierungsziele der wirtschaftlich schwächeren Mitgliedsländer. Technisch-wirtschaftliche Konvergenz zu den wirtschaftlich führenden Ländern hatte in der jeweiligen nationalen Planung eine höhere Priorität als die optimale Arbeitsteilung zwischen den Ländern. Dies bedeutet aber auch, dass viele Güter in vielen Ländern und mit jeweils niedrigen Stückzahlen hergestellt worden sind. Skaleneffekte konnten so nicht realisiert werden, Innovationskosten wurden dupliziert und mussten auf geringe Produktionszahlen umgelegt werden. Auch infolgedessen nahm der technologische Rückstand gegenüber dem Westen zu und die Ostblockstaaten hatten zunehmend ökonomische Anreize, mit dem Westen Handel zu treiben. Darüber hinaus erschien den Planern in einem Ostblockstaat die Lieferzusagen aus einem anderen Ostblockstaat sehr unsicher - würden die notwendigen Mengen rechtzeitig und in gewünschter Qualität zur eigenen Planerfüllung zur Verfügung stehen? Lieferungen aus dem nichtsozialistischen Wirtschaftsgebiet erschienen zuverlässiger. Selbst die Planer im Ostblock orientierten sich an der ökonomischen Vorteilhaftigkeit für das eigene Land. Mit dem Zusammenbruch des Ostblocks brach auch der RGW zusammen, weil sich nun jedes Land vollständig an den eigenen ökonomischen Interessen bei der Gestaltung der internationalen Wirtschaftsbeziehungen orientieren konnte. 
Die nationale wirtschaftliche Vorteilhaftigkeit sowie über ökonomische Kalküle hinausgehende innen- und außenpolitische Überlegungen spielen bei der Gestaltung einer Wirtschafts- oder Währungsgemeinschaft eine wichtige Rolle. So wie sich ökonomische und politische Faktoren im Zeitablauf wandeln können, sollte sich auch die Struktur einer Wirtschafts- und Währungsunion im Zeitablauf verändern.

Eine wichtige Strukturüberlegung bei der Schaffung des Euro war es, die Herrschaft der Bundesbank über die anderen westeuropäischen nationalen Zentralbanken zu begrenzen. Eine andere Überlegung betraf die Einbettung der durch die Vereinigung gewachsenen politischen Macht Deutschlands in europäische Institutionen. Aus deutscher Sicht stand einerseits die Zustimmung der Alliierten zur Wiedervereinigung auf dem Spiel. Andererseits war die ökonomische Bedeutung der europäischen Partnerländer beachtlich. Im Jahre 1991 wickelte die Bundesrepublik Deutschland beispielsweise rund die Hälfte ihres Außenhandels mit Ländern ab, die damals dem europäischen Währungsraum angehörten. Im Jahre 2012 entfielen auf Länder der Eurozone nur noch rund 38 Prozent des deutschen Außenhandels. Dabei hat Deutschland besonders von der europäischen Integration profitiert. Einerseits haben deutsche Firmen ihre Produktionsgeographie deutlich verändert, indem viele Produktionsschritte nach Osteuropa verlagert worden sind, das heißt deutsche Unternehmen haben die Allokation ihrer Ressourcen optimiert. ${ }^{14}$ Diese Optimierung der Produktionsstruktur hat ebenso zur Stagnation der Lohnstückkosten in Deutschland beigetragen wie die gewerkschaftliche Lohnzurückhaltung und die Arbeitsmarktreformen der Regierung Schröder. ${ }^{15}$ Gefördert wurden die Produktionsverlagerung nach Osteuropa und der innereuropäische Handel durch die Schaffung des Euro, weil dadurch Abwertungsrisiken entfallen sind, sowie durch die Osterweiterung der Europäischen Union. Die Abwesenheit von innereurozonalen Wechselkursrisiken hat auch die Kapitalströme zwischen den Euroländern beflügelt und zu einem Zinsrückgang in den Peripheriestaaten geführt. Ausgaben für langlebige Konsumgüter und für Wohnungsbauinvestitionen konnten in Südeuropa nun günstiger finanziert werden. Die wachsende Güternachfrage führte zu wachsender Nachfrage nach Arbeitskräften und zu steigenden Löhnen. Infolgedessen hat sich die Lohnstückkostenentwicklung in Italien, Spanien, Frankreich und anderen Peripherieländern seit dem Jahre 2000 von der deutschen Entwicklung abgekoppelt. ${ }^{16}$ Spiegelbildlich zur deutschen Entwicklung drehten die Handelsbilanzen dieser Länder ins Minus. ${ }^{17}$ Diese

14 Vgl. dazu Hans-Werner Sinn, Die Basar-Ökonomie. Deutschland - Exportweltmeister oder Schlusslicht? Berlin 2005.

15 Zwischen 1995 und 2008 stagnierten die Lohnstückkosten in Deutschland. Vgl. Paolo Guerrieri u. Piero Esposito, Intra-European Imbalances, Adjustment, and Growth in the Eurozone, in: Oxford Review of Economic Policy 28. 2012, S. 532-550, hier S. 538.

16 Ebd.

17 Ebd., S. 535. 
gegenläufige Entwicklung betraf jedoch nicht nur die Entwicklung innerhalb der Eurozone, sondern auch die Entwicklung gegenüber dem Rest der Welt. Vor Einführung des Euro hatten die Nord- und Südländer gegenüber dem Rest der Welt, betrachtet man sie jeweils als ein Block, näherungsweise ausgeglichene Zahlungsbilanzen. Nach Einführung des Euro erwirtschaftete der Norden gegenüber dem Rest der Welt große Überschüsse, während der Süden ebenso große Defizite generierte. Die Tatsache, dass sich Nordüberschüsse und Süddefizite gegenüber dem Rest der Welt ausglichen, führte dazu, dass sich der Wechselkurs des Euro gegenüber den anderen Währungen nicht veränderte. Gleichwohl wäre eine Anpassung notwendig. Vor Einführung des Euro hätten die Nordwährungen aufgewertet, die Südwährungen abgewertet werden müssen und die Handelsbilanzen innerhalb der Eurozone hätten sich ebenfalls ausgeglichen. Nach Einführung des Euro erfolgte diese Wechselkurskorrektur nicht, so dass die deutsche Exportwirtschaft von der wachsenden Fehlbewertung ihrer Handelswährung profitierte. ${ }^{18}$ Die Herrschaft der Bundesbank über die europäische Währungspolitik wurde in der Epoche des Euro durch die Herrschaft der deutschen Exportwirtschaft abgelöst. Auf die Übermacht der Bundesbank reagierten die anderen europäischen Länder mit regelmäßigen Abwertungen. Nach Einführung des Euro begegnete man der Herrschaft der deutschen Exportwirtschaft im europäischen Ausland bis zur Weltfinanzkrise mit Kreditaufnahme. Die mit der Einführung des Euro in den neunziger Jahren und bis 2007 immer stärker in Richtung der niedrigen deutschen Zinsrate fallenden Kosten für staatliche Kredite der Euroländer boten die Möglichkeit, die sinkende Wettbewerbsfähigkeit zumindest kurzfristig nicht in Wohlstandsverluste münden zu lassen - um den Preis einer immer höheren privaten und öffentlichen Verschuldung. Als ab September 2008 an den Finanzmärkten immer höhere Risikoaufschläge von den südeuropäischen Ländern verlangt wurden, drohte deren Zahlungsunfähigkeit, die nur durch Ausfallgarantien und Kredite der Euroländer und der Europäischen Zentralbank verhindert werden konnten. Begründet wurde dies mit der notwendigen Erhaltung der Stabilität des internationalen Finanzsystems und der Bedeutung des Euro für das europäische Einigungsprojekt.

Die Garantien und Kredite waren aber nicht umsonst zu haben. Vielmehr waren damit strikte Auflagen der Begrenzung staatlicher Ausgaben durch Kürzungen von Renten, Gehältern und Sparmaßnahmen in quasi allen staatlichen Ausga-

18 Martin Berka u. Micheal B. Devereux führen aus, dass die Währung in Griechenland um 13 Prozent, in Portugal um 16 Prozent, in Italien um 7 Prozent und in Spanien um 5 Prozent abgewertet werden müsste, um ihren realen Wechselkurs an den angemessenen Wert anzupassen. Da eine Abwertung innerhalb der Eurozone unmöglich ist, muss entweder die Produktivität entsprechend steigen - und zwar schneller als im Durchschnitt der Eurozone - oder die Produktionskosten (insbesondere die Löhne) müssen entsprechend gekürzt werden bzw. langsamer wachsen als im Durchschnitt der Eurozone, vgl. dies., Trends in European Real Exchange Rates, in: Economic Policy 28. 2013, S. 193 - 242. 
benbereichen verbunden. Diese interne Abwertung durch Lohn- und Kostensenkungen bedeutet eine seit der Wirtschaftskrise der dreißiger Jahre unbekannte Austeritätspolitik. Mit den entsprechenden Folgen: Die Maßnahmen der binnenwirtschaftlichen Konsolidierung führten zu Arbeitslosigkeit, Einkommensverlusten und Wachstumseinbußen. In der Folge kam es zu Bürgerprotesten und der Stärkung populistischer Kräfte in Griechenland ebenso wie in Italien. Zu beobachten ist auch ein aufkommender Neonationalismus, der seinen symbolischen Ausdruck etwa in Zeitungsüberschriften in Deutschland findet, in denen die „Faulheit“ und „Verschwendung“ südeuropäischer Länder karikiert werden. Umgekehrt tragen protestierende Griechen Plakate mit der deutschen Bundeskanzlerin als Hitler mit sich.

Dies alles macht fraglich, ob nur das verbissene Festhalten an der Währungsunion den Zerfall der Europäischen Union und des europäischen Einigungsprozesses verhindert oder ob nicht gerade umgekehrt der Versuch, die gemeinsame Währung zu retten, diesen politischen Zerfall hervorruft. Das System von Bretton Woods wie auch das Europäische Währungssystem sahen die Möglichkeit der abgestimmten Abwertung von Währungen vor, was verhinderte, dass den Ländern mit weniger wettbewerbsfähigen Ökonomien von außen Reformen aufgezwungen werden mussten. Freilich gingen die Abwertungen auf Kosten z. B. der deutschen Exportindustrie. Will man den Euro als Projekt nicht aufgeben, so wird man sich bald fragen müssen, ob den Ungleichgewichten zwischen den europäischen Volkswirtschaften nicht mit anderen Maßnahmen - beispielsweise europäischen Transferzahlungen oder unbegrenzten Krediten der Europäischen Zentralbank - entgegengetreten werden muss. Da die Schuldnerländer über eine Mehrheit in den europäischen Institutionen verfügen, dürfte dies der innenpolitisch opportune Weg sein.

Im Frühjahr 2013 ist es unmöglich, Prognosen über das weitere Schicksal des Euro anzustellen. Zumindest sollte man darauf verzichten, wenn man nicht ebenso falsch liegen will wie Helmut Kohl mit seinen optimistischen Prognosen, die er zu Beginn der Einführung des Euro als Bargeld anstellte. Dagegen ergibt es sehr viel Sinn, sich die Geschichte von Währungskrisen genauer anzusehen. Hierin sieht das vorliegende Themenheft sein zentrales Anliegen.

Prof. Dr. Jens Beckert, Max-Planck-Institut für Gesellschaftsforschung, Paulstraße 3, D-50676 Köln

E-Mail: beckert@mpifg.de

Prof. Dr. Carsten Burhop, Universität Wien, Historisch-Kulturwissenschaftliche Fakultät, Institut für Wirtschafts- und Sozialgeschichte, Universitätsring 1, A-1010 Wien

E-Mail: carsten.burhop@univie.ac.at 